\title{
Dynamic behaviour of a planar micro-beam loaded by a fluid-gap: analytical and numerical approach in a high frequency range, benchmark solutions
}

\author{
Antonin Novak ${ }^{1 *}$, Petr Honzik ${ }^{2}$, Michel Bruneau ${ }^{1}$ \\ ${ }^{1}$ Laboratoire d'Acoustique de I'Université du Mans, LAUM - UMR 6613 CNRS, Le Mans Université, Avenue Olivier \\ Messiaen, 72085 LE MANS CEDEX 9, France \\ ${ }^{2}$ Czech Technical University in Prague, Faculty of Transportation Sciences, Prague, Czech Republic \\ *antonin.novak@univ-lemans.fr, *https://ant-novak.com
}

\begin{abstract}
Miniaturized vibrating MEMS devices, active (receivers or emitters) or passive devices, and their use for either new applications (hearing, meta-materials, consumer devices, ...) or metrological purposes under non-standard conditions, are involved today in several acoustic domains. More in-depth characterisation than the classical ones available until now are needed. In this context, the paper presents analytical and numerical approaches for describing the behaviour of three kinds of planar micro-beams of rectangular shape (suspended rigid or clamped elastic planar beam) loaded by a backing cavity or a fluid-gap, surrounded by very thin slits, and excited by an incident acoustic field. The analytical approach accounts for the coupling between the vibrating structure and the acoustic field in the backing cavity, the thermal and viscous diffusion processes in the boundary layers in the slits and the cavity, the modal behaviour for the vibrating structure, and the non-uniformity of the acoustic field in the backing cavity which is modelled in using an integral formulation with a suitable Green's function. Benchmark solutions are proposed in terms of beam motion (from which the sensitivity, input impedance, and pressure transfer function can be calculated). A numerical implementation (FEM) is handled against which the analytical results are tested.
\end{abstract}

The archived file is not the final published version of the article A. Novak, P. Honzik \& M. Bruneau (2017), "Dynamic behaviour of a planar micro-beam loaded by a fluid-gap: Analytical and numerical approach in a high frequency range, benchmark solutions", Journal of Sound and Vibration. Vol. 401(2017), pp. 36-53.

The definitive publisher-authenticated version is available online at https://doi.org/10.1016/j.jsv.2017.04.026, Readers must contact the publisher for reprint or permission to use the material in any form. 


\section{Introduction}

In the past decades, much efforts has been put into miniaturizing acoustic elements (tubes, slits, cavities, membranes, ...) used in acoustic devices (absorbers, filters, transducers, ...) in order to reduce the sizes of these devices, to improve their properties or create new ones, or to lower their manufacturing cost (MEMS devices are more particularly concerned). More specifically, there has been increasing interest in designing miniaturized vibrating devices (using MEMS processes) [1], active (receivers or emitters) or passive devices, and in using them for both new applications which involve several fields of acoustics (hearing, meta-materials, consumer devices,...) and metrological purposes under non-standard conditions, namely high frequency ranges (typically up to $500 \mathrm{kHz}$ ), gas mixtures, and various static pressures and temperatures [2]. The miniaturisation and these new applications and purposes require deeper characterisations (analytical, numerical, and experimental) as the classical ones available until now. In this context, much of the analytic work concerns devices with two-dimensional (circular or square) micro-structures (membranes or thin plates) for which researches into the effective design have been carried out [3, 4].

The present article is concerned with the analytical and numerical approaches of the behaviour of a onedimensional device, a planar micro-beam of rectangular shape whose length is much greater than the width and whose thickness is much lower than the width, loaded by a backing cavity or a fluid-gap (squeeze film), surrounded by very thin slits, and excited by an incident acoustic field (assumed to be uniform on the plate). The micro-beam is either a suspended rigid one-dimensional planar beam or a thin elastic one-dimensional plate clamped to one end, the other (free) end being eventually loaded by a punctual mass. The suspended rigid planar beam, which oscillates perpendicularly to its plane, is either fixed at both ends on non-rigid walls (spring-like boundaries) or attached to one end of a flat spring of negligible mass (the other end of the spring being free) (Fig. 1). The slits surrounding the beam permit both to ensure the static pressure equilibrium on both faces of the beam (role played by the vent-holes in the classical devices) and to fit to some extent the damping of the beam, along with avoiding the design of a complex suspension (note that additional damping due to non-linear phenomena which take place around the sharp edges is neglected owing to the small amplitude of the oscillations). It is worth noting that a direct coupling between the incident acoustic field and the acoustic field in the backing cavity could take place significantly, through the slits.

The literature abounds with many papers in which topics involving flexible microstructures (oscillating masses or beams, vibrating plates, membranes, or beams) and squeeze-films are of principal focus. The papers deal extensively with the viscous damping in squeeze-films which are the most significant mechanism of energy dissipation, because it is essential to understand this damping mechanism to optimise the design of these MEMS devices. The most important parameter is the Knudsen number $K_{n}$, defined as the ratio of the molecular mean free path $\lambda_{m f p}$ (at the static pressure considered) to the characteristic length of the acoustic flow, namely here the thickness $h_{g}$ of squeeze-film $\left(K_{n}=\lambda_{m f p} / h_{g}\right)$, where the mean free path $\lambda_{m f p}$ at the static pressure considered $P_{a}$ is linked to the mean free path $\lambda_{m f p}^{(0)} \simeq 65 \mathrm{~nm}$ at the atmospheric pressure $P_{0}$ by $\lambda_{m f p}=\left(P_{0} / P_{a}\right) \lambda_{m f p}^{(0)}$. Based on the value of this number, the flow inside the squeeze-film can be considered or not as continuous fluid flow. Many MEMS devices operate at very low pressure with a very small squeeze-film thickness $\left(K_{n}>10\right)$ so that the continuous fluid flow regime assumption cannot be assumed (free molecular flow regime treated in using Boltzmann equation) $[5,6,7]$. Note that the frequency involved in these papers is not much lower than the average molecular collision frequency, ranging typically from $1 \mathrm{MHz}$ to $100 \mathrm{GHz}$, while in the present paper the frequency is lower than $1 \mathrm{MHz}$. The MEMS devices considered in the present paper operate at atmospheric pressure with a fluid gap thickness larger than ten micrometers $\left(K_{n}<0.005\right)$; therefore the fluid can be treated as continuum (and it is assumed to be Newtonian), because the mean free path is much lower than the thickness of fluid-gap, with non-slip conditions at the walls (plate or beam). Note that in the intermediate regimes, respectively slip flow regime $\left(0.005<K_{n}<0.1\right)$ and transitional flow regime $\left(0.1<K_{n}<10\right)$, the validity of the modelling assuming continuous flow can be extended in using the 
so-called "effective viscosity coefficient" which depends on the Knudsen number [8].

In the review provided in a paper published in 2003 [9, and references 6 to 23 therein], the authors point out that in much of the literature involving the situations where continuous fluid flow can be assumed, the majority of the models treat the microplate as a rigid structure, and that only few model account for flexibility of the microstructure. Moreover, in this last situation, the coupling between the fluid-film and the elastic plate is not fully modelled and in the two-dimensional situations the lateral pressure variation is neglected when the flexible microstructure structure is considered as a one-dimensional one. These analytical approaches, while appropriate to addressing specific situations, have shortcomings which prevent in several situations from obtaining accurate results, tend to preclude extension to more sophisticated problems, and inhibit more advance insight. Therefore, in the same paper, the authors present an analytical approach based on modal expansions for both the fluid-film and a flexible two-dimensional microstructure (elastic plate), which account for the strong coupling between both, assuming several boundary conditions for the plate (free or clamped edges) and for the fluid-film (pressure or displacement vanishing). Then the authors use a perturbation method to treat the problem of matching both expansions near the boundaries and they solve numerically the final system of equations. In a paper published later on (in 2009) [10], which focuses attention on the fluid-film, an exact solution for the acoustic movement in the fluid film, which account for both viscosity effect in the boundary layers for the complex velocity field and complex behaviour varying from adiabatic to isothermal behaviour for the temperature field, is provided, the results being given in assuming open or closed border conditions, rigid and ideally thermal conducting plates (the moving and non-moving ones). Effects of viscosity and temperature variations on the movement of the fluid-film are also accounted for in a recent paper [11] (2016) which consider an oscillating rigid beam (as one of the beams considered below in the present paper) whose the movement is detected with a nano-gauge (microphone).

Regarding the analytical solutions for the squeeze-films coupled with the micro-beams or micro-plates, when continuous fluid flow can be assumed $\left(K_{n}<0.005\right)$, in the most advanced literature $[9,10,11$, for example] the following approaches are commonly used: i- the basic equations include the Navier-Stokes equation reduced to the linear Reynolds equation for a laminar flow (assuming pressure field independent of the coordinate normal to the fluid layer), the conservation of mass equation, the Fourier equation for conduction of heat (often reduced to the adiabatic or isothermal hypothesis), ii- the boundary conditions include non-slip condition and ideally heat conducting plate or beam, and always Dirichlet (pressure null) or Neumann (velocity null) conditions at the surrounding of the fluid gap. Beyond both the equivalent circuit model [8] providing a procedure for the dynamical analysis of the system in the lower frequency range (which account for the coupling between the vibrating structure and the acoustic field in the squeeze-film) and the exact solution [10] for the velocity and the temperature fields in the fluid-film in the highest frequency range considered (up to $10 \mathrm{kHz}$ ) which include the thermal effects (the vibrating structure being considered as a given source of energy), the most common technique employed to solve the coupled (structure/fluidgap) problems relies on the sets of the appropriate eigenmodes of both the displacement field of the vibrating wall and the pressure field in the fluid gap behind it $[9,12,13]$, the solutions accounting usually for the strong coupling between both $[9,13]$.

In reference [12] the coupling is neglected. In references $[9,13]$, the authors point out that, when the coupling is accounted for, matching these expansions near the edge of both the plate and the fluid-gap is a delicate task, because the mode shapes does not fit together near the edge where the acoustic field can change sharply. The techniques used to overcome these difficulties are not very simple to handle: in reference [9] the authors make use of a perturbation analysis and solve equations in using a finite-element method (assuming Dirichlet or Neumann boundary conditions), while in reference [13] the modal sum for the acoustic field is expressed as an expansion on the eigenmodes of the wall through a mixed boundary condition at the edge, leading to an equivalent network.

In order to overcome these difficulties, the analytical technique used herein, builds upon a previous work ([4] two-dimensional device), relies on a simple integral formulation (convolution product, Eq. (23) below) which involves 


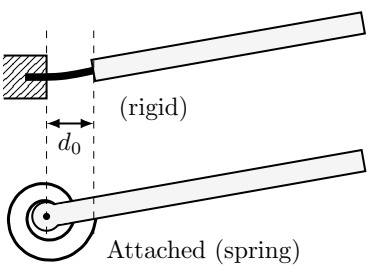

(a)

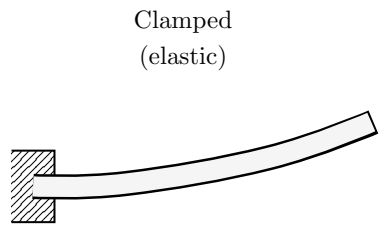

(b)
Elastically supported

(rigid)

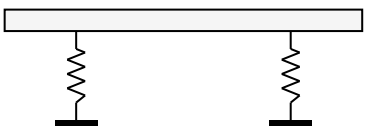

(c)

Figure 1. Sketch of the mechanical system consisting of a rigid planar beam attached to one end of a flat spring (a), a thin elastic one-dimensional planar beam clamped to one end (b), a suspended rigid planar beam (c).

a suitable Green's function for the acoustic field inside the fluid-gap, the displacement field of the structure being expressed as an expansion over orthonormal appropriate eigenfunctions (see Appendix A.3). This Green's function (Eq. (25) below) presents two major advantages: it is expressed in using simple functions (here trigonometric complex functions) avoiding modal expansion and it allows accounting for any complex boundary conditions at the periphery that cannot be achieved by a set of eigenfunctions. This is relevant whenever acoustic small elements are connected on the boundaries; this is important for current applications (mentioned above at the beginning of this introduction). Moreover, the analytical approach used herein to solve the displacement field of the structure and the acoustic field in the fluid gap, coupled together, account for not only the effects of the viscous boundary layers but also those of the thermal boundary layers. This is relevant here for the thickness of the fluid gap (around 100 micrometers) and the frequency range (up to $1 \mathrm{MHz}$ ) considered, because the compressibility of the gas is a complex function, tending to the real isothermal one in the lower frequency range and to the real adiabatic one in the higher frequency range (see Appendix A.4). Finally, the three prototype problems mentioned above (which could be typically encountered in the devices designed), namely thin elastic planar beam clamped at one end or rigid planar beam attached to one or two springs, with slits around them, loaded by viscous and thermal conducting thin fluid-films, are solved analytically. They provide the motions of the beams and the acoustic field in the backing cavity, leading to sensitivity, input impedance, or acoustic pressure transfer function, depending on the device considered. A numerical implementation (FEM) is handled against which the analytical results are tested.

To sum up, on the one hand the paper involves three kinds of beams and, on the other hand it involves, among specific modelling for each kind of beam, a formulation which gives a prominent role to both the viscous and thermal boundary layer effects (in the slits and in the backing cavity), and to the integral formulation with a suitable Green's function (which satisfy any kind of boundary conditions) used to express the acoustic field in the closed backing cavity. Thus, the paper is divided mainly in three parts: after the presentation of the three kinds of devices (section 2.1) and the analytical approach to describe both the effects of the slits and the pressure field in the backing cavity (sections 2.2 and 2.3 respectively), equations governing the motion of each kind of beam and corresponding analytical solutions are presented in sections 3.1 to 3.3, while details on the analytical results are relegated to A.1 and A.2. Finally, after a short presentation of the numerical (FEM) method used and before concluding, the last section 4 presents respectively several analytical and numerical results, and comparison between them. Appendix A.3 and A.4 provide details respectively on the orthogonality (and the completeness) of the eigenfunctions of the elastic beam and on the thermal behaviour of the fluid-gap. 


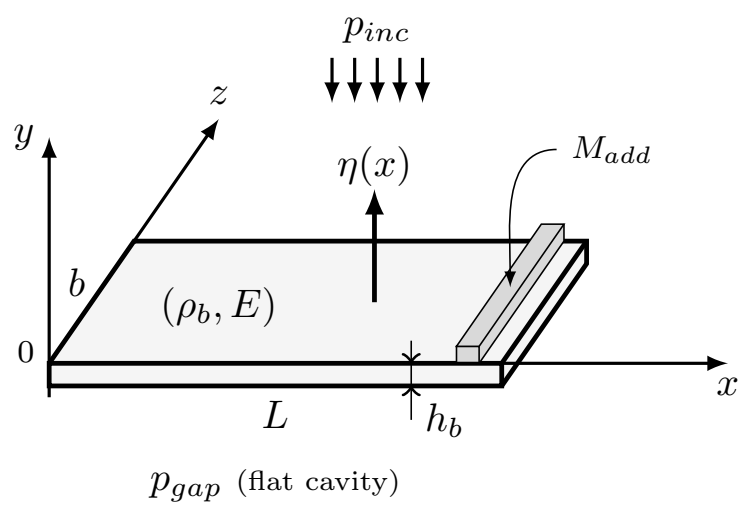

(a)

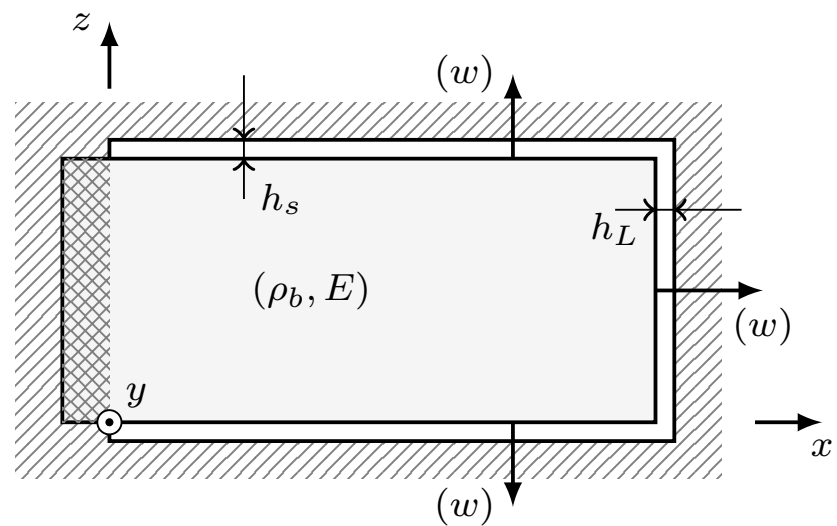

(b)

Figure 2. Sketch of the rectangular planar beam (a) and the slits between the beam and the wall (b).

\section{The device, the fluid motions inside it}

\subsection{The device: micro-beam, slits, backing cavity}

The so-called one-dimensional device considered is a planar micro-beam of rectangular shape (one-dimensional plate) of density $\rho_{b}$ and either rigid or elastic (Young modulus $E$ ), whose the length $L$ is much greater than the width $b$ and whose the thickness $h_{b}$ is much lower than the width $\left(h_{b} \ll b\right)$ (Fig. 2(a)). It is loaded by a backing cavity or fluid-gap (pressure variation $p_{\text {gap }}$ ), surrounded by very thin slits of thicknesses $h_{s}$ and $h_{L}$ (Fig. 2(b)), and excited by an incident acoustic field $p_{\text {inc }}$ (assumed to be uniform over the plate). The beam is either a suspended rigid plate (Figs. 1(a) and 1(c)) or a thin elastic one-dimensional plate clamped to one end (Fig. 1(b)). The suspended rigid plate, which oscillate normally to its plane in the $y$-direction, is either fixed at both ends on non-rigid walls (spring-like boundaries) (Fig. 1(c)) or attached to one end of a flat spring of negligible mass (the other end of the spring being clamped) (Fig. 1(a)), the other end of the beam being eventually loaded by a punctual mass $M_{\text {add }}$. The flat spring, which is commonly designed, could be modelled as follows: the end of the beam is attached to its rigid surrounding frame with a pivot link, and at a small distance from this end a restoring force and a damping force are applied respectively by a spring and a dashpot (that provide viscous damping). The cartesian coordinate system used $(x, y, z)$ has its origin at the attach end $x=0$ of the beam, the $\mathrm{O} x$-axis is directed towards the other end of the beam, the $\mathrm{O} y$-axis is perpendicular to the plate beam and outwardly directed, and the $\mathrm{O} z$-axis is oriented as indicated in Fig. 2. The local $w$-axes have their origins on the edges of the beam and are directed towards the walls surrounding the beam.

The behaviour of both the particle velocity and the pressure variations in the slits and in the backing cavity is investigated analytically in the next sections (2.2) and (2.3) respectively. This makes possible the detailed analytic modelling of the behaviour of the beam, which includes the effects of the slits and of the backing cavity, presented beyond in sections (3.1) to (3.3).

\subsection{Slits effects: viscous forces acting on the beam, acoustic pressure coupling through the slits}

Consider the thin slits trapped between the planar beam (of thickness $h_{b}$ ) and the walls with an outward (i.e. directed towards the wall) local $w$-axis (its origin being set on the edges of the beam) (Fig. 3) and let $h_{s}$ and $h_{L}$ designate the thickness of the lateral slits (along the side wall of the beam) and the thickness of the slit at the end of the beam, respectively (Fig. 2(b)).

Assume that a viscous fluid (of density $\rho_{0}$, shear viscosity $\mu$, and speed of sound $c_{0}$ ) oscillates back and forth 


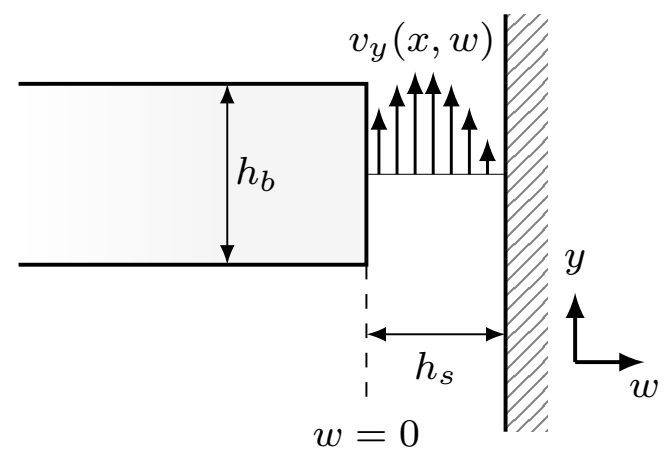

Figure 3. Sketch of the peripheral slit between the beam and the wall.

inside the slits due to the pressure gradient between the pressure field inside the backing cavity $p_{\text {gap }}(x)$ and the external pressure field $p_{\text {inc }}$ (incident uniform pressure field), and that this incident field $p_{\text {inc }}$ is harmonic (given that the time dependence is $\left.e^{i \omega t}\right)$. The viscous force along the $y$-axis $d F_{s, y}(x)$ acting on an elementary surface (area $h_{b} d x$ ) of any lateral side (set at $w=0$ ) of the beam and the viscous force $F_{L, y}$ acting on the surface (area $\left.h_{b} b\right)$ of the end $x=L$ of the beam takes respectively the following forms

$$
\begin{gathered}
d F_{s, y}(x)=\mu h_{b} d x\left(\frac{\partial v_{y}(x, w)}{\partial w}\right)_{w=0}, \\
F_{L, y}=\mu h_{b} b\left(\frac{\partial v_{y}(L, w)}{\partial w}\right)_{w=0},
\end{gathered}
$$

where equations for the particle velocity profile $v_{y}(x, w)$ include on the one hand the diffusion equation [14].

$$
\frac{\partial^{2} v_{y}(x, w)}{\partial w^{2}}+k_{v}^{2} v_{y}(x, w)=\frac{1}{\mu} \frac{\partial p}{\partial y}
$$

and on the other hand, assuming non-slip conditions on the walls $\left(w=h_{s}\right.$ or $\left.w=h_{L}\right)$ and on the lateral surface $w=0$ of the beam respectively, the boundary conditions

$$
\begin{cases}v_{y}(x, w)=0, & w=h_{s} \\ v_{y}(x, w)=i \omega \eta(x), & w=0,\end{cases}
$$

with

$$
k_{v}=\frac{1-i}{\sqrt{2}} \sqrt{\omega \frac{\rho_{0}}{\mu}}
$$

$\eta(x)$ denoting the displacement field of the planar beam.

This system of equations $(3,4)$ is satisfied by

$$
v_{y}(x, w)=\left[i \omega \eta(x)+\frac{1}{i \omega \rho_{0}} \frac{\partial p}{\partial y}\right] \cos \left(k_{v} w\right)-\left[i \omega \eta(x) \cot \left(k_{v} h_{s}\right)-\frac{1}{i \omega \rho_{0}} \frac{\partial p}{\partial y} \tan \left(\frac{k_{v} h_{s}}{2}\right)\right] \sin \left(k_{v} w\right)-\frac{1}{i \omega \rho_{0}} \frac{\partial p}{\partial y} .
$$

Then, invoking these expressions for the particle velocity distribution across the thickness of the slits, equations (1) and (2) lead readily to

$$
\begin{gathered}
d F_{s, y}(x)=\left[-\frac{1}{2} K_{v, s} h_{s}\left(p_{\text {inc }}-p_{\text {gap }}(x)\right)+i \omega \eta(x) \Pi_{s}\right] d x \\
F_{L, y}=\left[-\frac{1}{2} K_{v, L} h_{L}\left(p_{\text {inc }}-p_{\text {gap }}(L)\right) b+i \omega \eta(L) \Pi_{L}\right] b
\end{gathered}
$$




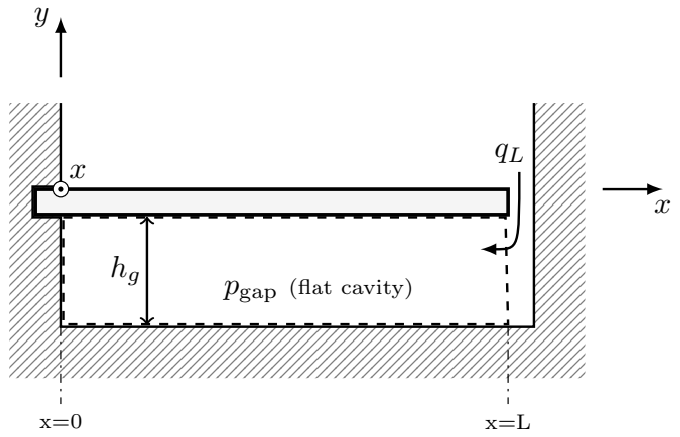

(a)

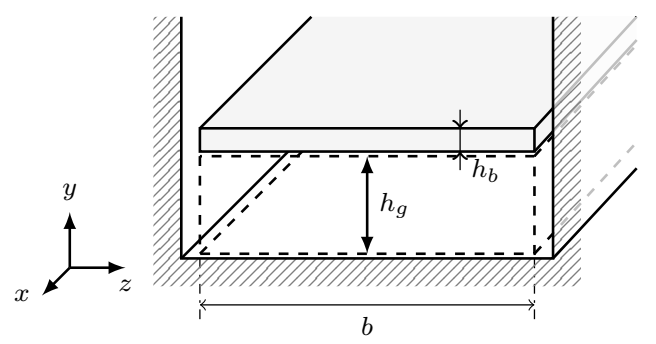

(b)

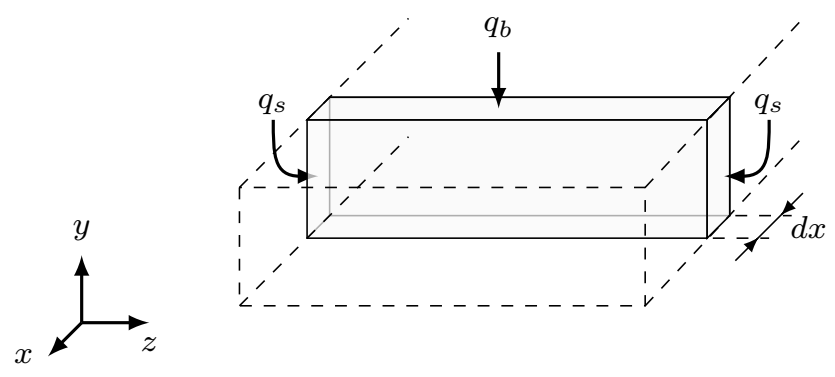

(c)

Figure 4. Sketch of the backing cavity (a), of its cross section (b), and of an elementary volume inside it (length $d x)(\mathrm{c})$.

after relying on the following approximation

$$
\frac{\partial p}{\partial y} \cong \frac{p_{\text {inc }}-p_{\text {gap }}(x)}{h_{b}},
$$

with

$$
\begin{gathered}
K_{v, a}=\frac{\tan \left(\frac{k_{v} h_{a}}{2}\right)}{\frac{k_{v} h_{a}}{2}}, \\
\Pi_{a}=-k_{v} \mu h_{b} \cot \left(k_{v} h_{a}\right)
\end{gathered}
$$

the subscript $a$ designating either $s$ or $L$.

It is worth noting that these forces $(7,8)$ involve the sum of two terms: the first one, proportional to the pressure difference between the pressure fields across the beam (acoustic pressure coupling through the slits), originates the velocity field inside the slit, whereas the second one involves the velocity of the beam which affects the viscous force on the sides and the end of it.

\subsection{Acoustic pressure in the fluid gap (backing cavity)}

Consider the thin backing cavity (of thickness $h_{g}$, width $b$, and length $L$ ) and an elementary volume (of thickness $h_{g}$, width $b$, and length $d x$ ) inside it, and let $q_{s}, q_{b}$, and $q_{L}$ designate the input volume velocity in this elementary volume of an element (length $d x$ ) of the lateral slits, of an element (length $d x$ ) of the beam, and of the slit at the end of the beam (when $x=L$ ), respectively (Fig. 4).

Assume that the fluid is not only viscous but also heat conducting (of thermal conductivity $\lambda_{h}$ and heat coefficient at constant pressure per unit of mass $C_{P}$ ), and that both the velocity field and the pressure field are uniform over 
the width $b$ of the fluid-gap inside the backing cavity (the pressure field being also uniform through the thickness $\left.h_{g}\right)$. The total input volume velocity $q(x)=q_{s}(x)+q_{b}(x)+q_{L}(x)$ takes the following form:

$$
q(x)=-2 \frac{h_{s} \bar{v}_{s, y}(x)}{h_{g} b}-\frac{i \omega \eta(x)}{h_{g}}-\frac{h_{L} \bar{v}_{L, y}}{h_{g}} \delta(x-L),
$$

where $\bar{v}_{s, y}$ and $\bar{v}_{L, y}$ denote the mean value of the velocity field (6) across the lateral slits and the slit at the end $L$ of the beam respectively

$$
\begin{gathered}
\bar{v}_{s, y}(x)=-\frac{1}{i \omega \rho_{0}} \frac{p_{\text {inc }}-p_{\text {gap }}(x)}{h_{b}} F_{v, s}+\frac{1}{2} i \omega \eta(x) K_{v, s}, \\
\bar{v}_{L, y}=-\frac{1}{i \omega \rho_{0}} \frac{p_{\text {inc }}-p_{\text {gap }}(L)}{h_{b}} F_{v, L}+\frac{1}{2} i \omega \eta(L) K_{v, L},
\end{gathered}
$$

with

$$
F_{v, a}=1-K_{v, a}
$$

the expression of $K_{v, a}$ being given by equation (10).

Then the acoustic pressure field $p_{\text {gap }}(x)$ is governed by the following equation of propagation [13]

$$
\left(\frac{\partial^{2}}{\partial^{2} x}+\chi_{0}^{2}\right) p_{\text {gap }}(x)=-\frac{i \omega \rho_{0}}{F_{v, g}} q(x)
$$

and is subjected to mixed boundary conditions at both ends $x=0, L$

$$
\left(\frac{\partial}{\partial x}-\zeta(0)\right) p_{\text {gap }}(0)=0, \quad\left(\frac{\partial}{\partial x}+\zeta(L)\right) p_{\text {gap }}(L)=0
$$

Invoking equations $(12-15)$, equation (16) can be written as:

$$
\left(\frac{\partial^{2}}{\partial^{2} x}+\chi^{2}\right) p_{\text {gap }}(x)=-U(x)
$$

with

$$
\chi^{2}=-2 \frac{F_{v, s}}{F_{v, g}} \frac{h_{s}}{h_{g} h_{b} b}+\frac{\omega^{2}}{c_{0}^{2}} \frac{\gamma-(\gamma-1) F_{h, g}}{F_{v, g}},
$$

where

$$
F_{h, g}=1-\frac{\tan \left(\frac{k_{h} h_{g}}{2}\right)}{\frac{k_{h} h_{g}}{2}},
$$

with

$$
k_{h}=\frac{1-i}{\sqrt{2}} \sqrt{\omega \frac{\rho_{0} C_{p}}{\lambda_{h}}}
$$

and

$$
U(x)=-\frac{i \omega \rho_{0}}{F_{v, g}} \frac{h_{L}}{h_{g}} \bar{v}_{L, y} \delta(x-L)+2 \frac{F_{v, s}}{F_{v, g}} \frac{h_{s}}{h_{g} h_{b} b} p_{\mathrm{inc}}+\frac{\rho_{0} \omega^{2}}{F_{v, g} h_{g}}\left[1+\frac{h_{s}}{b} K_{v, s}\right] \eta(x),
$$

which depends on $x$ only through the coupled displacement field of the beam (see comments on Eq. (19) in Appendix A.4).

The viscous factor $F_{v, g}$ arises from the mean value, across the thickness of the fluid-gap, of the velocity profiles along the $x$-axis, expressed as mentioned in the previous section (Eq. 6) when both boundary conditions are homogeneous (i. e. when the backing wall and the beam are at rest in the $x$-direction). Similarly, the thermal factor $F_{h, g}$ results from the same formalism as the one that leads to the factor $F_{v, g}$ (diffusion process in the thermal boundary layers with isothermal conditions on the walls). 
With the inhomogeneous source function $U(x)$ in the right hand side of Eq. (18), and with both the pressure variation $p_{\text {gap }}$ and a Green's function $G\left(x, x_{0}\right)$ satisfying the same admittance boundary conditions (17) at the ends $x=0$ and $x=L$ of the backing cavity, the solution for $p_{\text {gap }}$ is given by

$$
p_{\text {gap }}(x)=\int_{0}^{L} G\left(x, x_{0}\right) U\left(x_{0}\right) d x_{0} .
$$

The Green function $G\left(x, x_{0}\right)$ which is the solution to the differential equation [the same operator as in the left-hand side of equation (18)]

$$
\left(\frac{\partial^{2}}{\partial^{2} x}+\chi^{2}\right) G\left(x, x_{0}\right)=-\delta(x)
$$

with the boundary conditions (17) at $x=0$ and $x=L$, is [15]

$$
G=\frac{-1}{\chi \sin \left(\chi L+\alpha_{0}+\alpha_{L}\right)} \cos \left(\chi x_{<}+\alpha_{0}\right) \cos \left(\chi\left(x_{>}-L\right)-\alpha_{L}\right)
$$

where $\left(x_{<}, x_{>}\right)$designate the smaller/larger of $x, x_{0}$ respectively, and where $\alpha_{0}$ and $\alpha_{L}$ are given by

$$
\zeta(0)=-\chi F_{v, g} \tan \left(\alpha_{0}\right), \quad \zeta(L)=-\chi F_{v, g} \tan \left(\alpha_{L}\right) .
$$

Given the Green's function displayed above, what follows is (23)

$$
p_{\text {gap }}(x)=\int_{0}^{x} G\left(x \equiv x_{>}, x_{0} \equiv x_{<}\right) U\left(x_{0}\right) d x_{0}+\int_{x}^{L} G\left(x \equiv x_{<}, x_{0} \equiv x_{>}\right) U\left(x_{0}\right) d x_{0} .
$$

The analysis can be readily adapted, furthermore, to include (for example) a small hole described by its input volume velocity as a function of the pressure variation at its entrance, which could be of practical interest. It may be noted that the integral involving the term $\left(i \omega \rho_{0} / F_{v, g}\right)\left(h_{L} / h_{g}\right) \bar{v}_{L, y} \delta(x-L)$ (Eq. 22) describes a boundary condition at $x=L$ expressed by

$$
\left(\frac{\partial}{\partial x}+\zeta(L)\right) p_{\text {gap }}(L)=\frac{i \omega \rho_{0}}{F_{v, g}} v_{x}(L)
$$

where (velocity flow conservation)

$$
b h_{g} v_{x}(L)=-b h_{L} \bar{v}_{L, y} .
$$

This result is verified by substituting successively the boundary condition (28) for the pressure variation and the boundary condition (17) for the Green's function into the integral (23), invoking equation (29):

$$
\begin{aligned}
& -\int_{0}^{L} G\left(x, x_{0}\right)\left[\frac{i \omega \rho_{0}}{F_{v, g}} \frac{h_{L}}{h_{g}} \bar{v}_{L, y} \delta(x-L)\right] d x_{0}=G(x, L)\left[\frac{i \omega \rho_{0}}{F_{v, g}} v_{x}(L)\right] \\
& =G(x, L) \frac{\partial p_{g}\left(x_{0}=L\right)}{\partial x_{0}}+p_{g}(L) \zeta(L) G(x, L)=G(x, L) \frac{\partial p_{g}\left(x_{0}=L\right)}{\partial x_{0}}-p(L) \frac{\partial G\left(x, x_{0}=L\right)}{\partial x_{0}}
\end{aligned}
$$

where the last expression is the 1-D integral representation of the boundary condition at $x=L$, as expected.

Note that, for the study of the dynamical behaviour of the system considered in this paper, we are left here with two coupled equations, namely equation (27) which relates the acoustic pressure inside the fluid-gap to both itself and the displacement field of the planar beam [that are included in functions (14) and (22)] and, conversely, equation which relates the displacement field of the planar beam to itself and to the acoustic pressure inside the fluid-gap. This last equation, which governs the displacement field of the planar beam, is presented in the next section, for each beam considered. 


\section{The motion of the beams}

\subsection{Rigid one-dimensional planar beam attached to one end of a flat spring of negligible mass}

The differential equation which governs the amplitude of the displacement field $\eta(x, t)=x \theta(t)$ (positive when directed along the $y$-axis) of the rigid planar beam of mass moment of inertia $J$, attach to one end of a flat spring of negligible mass, of force moment $C_{b}$ and damping coefficient $D_{b}$ (the upper figure $1(\mathrm{a})$ ), can be written as:

$$
\left[-\omega^{2} J+i \omega D_{b}+C_{b}\right] \theta=b \int_{0}^{L}\left(p_{\text {gap }}(x)-p_{\text {inc }}\right) x d x-2 \int_{0}^{L}\left(\frac{d F_{s, y}}{d x}\right) x d x-L F_{L, y},
$$

where the three external moments in the right hand side are descriptive respectively of the acoustic pressure fields on both sides of the beam, of the lateral slits (7), and of the slit at the end $L$ of the beam (8), with

$$
\int_{0}^{L} p_{\text {inc }} x d x=\frac{L^{2}}{2} p_{\text {inc }}
$$

because the harmonic incident pressure field $p_{\text {inc }}$ is assumed to be uniform over the planar beam. The sketch of the lower figure 1(a) shows an equivalent suspension where the end $(x=0)$ is attached to its surrounding frame with pivot link, restoring force and damping force being applied on the beam near its end.

It follows from expression (27) of $p_{\text {gap }}$ and (22) of $U$, after a lengthy but straightforward calculation (see the procedure in Appendix A.1), that the angle $\theta$ is expressed by:

$$
\theta=-p_{\text {inc }} \frac{\left(b-K_{v, s} h_{s}\right)\left(\frac{L^{2}}{2}+A_{1} B_{1}+A_{2}\right)-\frac{1}{2} K_{v, L} h_{L} b L\left(1+B_{1}\right)}{-\omega^{2} J+i \omega\left(D_{b}+2 \Pi_{s} \frac{L^{3}}{3}+\Pi_{L} b L^{2}\right)+C_{b}+\left(b-K_{v, s} h_{s}\right)\left(A_{1} B_{2}+A_{3}\right)-\frac{1}{2} K_{v, L} h_{L} b L B_{2}},
$$

where the frequency dependent integration constant $A_{1}, A_{2}, A_{3}, B_{1}$, and $B_{2}$ are given by Eqs. (A.4)-(A.8) and where $\Pi_{s}$ and $\Pi_{L}$ are defined in Eq. (11).

\subsection{Thin elastic one-dimensional flat beam clamped at one end and free at the other end, with an added mass at the free end}

The thin elastic one-dimensional flat beam considered in this section is clamped at its end $(x=0)$ and free at its end $(x=L)$ (figure $1(\mathrm{~b})$ ). The following notations are used, among others (see previous sections): $\mu_{L}$ mass per unit length of the beam, $E$ Young modulus, $\left(I / \Sigma=(1 / \Sigma) \int y^{2} d \Sigma\right)$ radius of gyration (where the integration is over the whole area of the cross section of the beam) which is here equal to $\left(h_{b}^{2} / 12\right)$ for the rectangular cross-section, and localised mass $M_{\text {add }}$ at the end $x=L$. The set of equations which govern the amplitude of the displacement field $\eta(x, t)$ (positive when directed along the y-axis) of the elastic beam, can be written classically as [16]:

$$
\begin{gathered}
{\left[E I \frac{\partial^{4}}{\partial x^{4}}-\mu_{L} \omega^{2}\right] \eta(x)=b\left[p_{\text {gap }}(x)-p_{\text {inc }}\right]-2 \frac{d F_{s, y}}{d x}-\left(F_{L, y}-M_{\text {add }} \omega^{2}\right) \delta(x-L), \quad 0<x<L,} \\
\frac{\partial^{3} \eta}{\partial x^{3}}=0 \quad \text { and } \quad \frac{\partial^{2} \eta}{\partial x^{2}}=0, \quad x=L, \\
\frac{\partial \eta}{\partial x}=0 \quad \text { and } \quad \eta=0, \quad x=0,
\end{gathered}
$$

where the three external forces in the right hand side of the equation are descriptive respectively of the acoustic pressure fields on both sides of the beam, of the lateral slits (7), of the slit at the end $L$ of the beam (8), and of the inertial force due to the localized mass $M_{\text {add }}$. Note that both the viscous force at the edge $x=L$ and the inertial force has been removed from the boundary condition involving the elastic force $E I \partial^{3} \eta(x=L) / \partial x^{3}$ [first equation 
(35)] and added to the right hand side of the propagation equation with a $\delta$-function. It is worth noting that, when dealing with a plate fixed on non-rigid walls, the force function $f_{L, y}$ could include the force from the boundary (a spring-like force for example) and that, at $x=0$, the same kind of boundary conditions as at $x=L$ could occur (giving an additional term $f_{0} \delta(x)$ in the right side of equation 34).

The construction of solutions of equations (34) to (36) in terms of eigenmodes of the 1-D planar beam makes use of modal wave functions that are solutions of the homogeneous equation

$$
\left[\frac{\partial^{4}}{\partial x^{4}}-K_{m}^{4}\right] \psi_{m}(x)=0
$$

and subject to the boundary conditions (35) and (36).

These orthonormal eigenfunctions (see Appendix A.3) have the form

$$
\psi_{m}(x)=\frac{1}{\sqrt{L}}\left\{\left[\cos \left(K_{m} x\right)-\cosh \left(K_{m} x\right)\right]+\beta_{m}\left[\sin \left(K_{m} x\right)-\sinh \left(K_{m} x\right)\right]\right\},
$$

with

$$
\beta_{m}=\frac{\sin \left(K_{m} L\right)-\sinh \left(K_{m} L\right)}{\cos \left(K_{m} L\right)+\cosh \left(K_{m} L\right)}=-\frac{\cos \left(K_{m} L\right)+\cosh \left(K_{m} L\right)}{\sin \left(K_{m} L\right)+\sinh \left(K_{m} L\right)} .
$$

and the corresponding eigenvalues are solutions of equation $\cos \left(K_{m} L\right) \cosh \left(K_{m} L\right)=-1$ whose the roots are approximately given by

$$
K_{m} L \cong \begin{cases}1.2 \frac{\pi}{2} & m=0 \\ (2 m+1) \frac{\pi}{2} & m=1,2,3, \ldots\end{cases}
$$

The forced vibrations of the plate beam, driven by the incident uniform time-periodic acoustic pressure $p_{\text {inc }}$ and loaded by both the acoustic pressure in the air-gap $p_{\text {gap }}(x)$ and the velocity fields in the surrounding slits, are expressed by an expansion on this set of orthogonal functions as follows:

$$
\eta(x)=\sum_{m} \xi_{m} \psi_{m}(x)
$$

with

$$
\xi_{m}=\frac{\left(b-K_{v, s} h_{s}\right)\left[\int_{0}^{L} p_{\text {gap }}(x) \psi_{m}(x) d x-p_{\text {inc }} \int_{0}^{L} \psi_{m}(x) d x\right]-\left[F_{L, y}-M_{\text {add }} \omega^{2}\right] \psi_{m}(L)}{E I K_{m}^{4}-\omega^{2} \mu_{L}+i \omega 2 \Pi_{s}}
$$

after relying on equations (7) and (8). Three terms in the right hand side, including those which involve $p_{\text {gap }}$, are related to the displacement $\eta$, so they involve the coefficients $\xi_{m}$ themselves. Therefore, it follows from expressions (27) of $p_{\text {gap }}$ and (22) of $U(x)$, after a lengthy but straightforward calculation (see the procedure in Appendix A.2), that the unknown coefficients $\xi_{m}$, which lead to $\eta(x)$ then to $p_{\text {gap }}(x)$, are solution of the following set of linear algebraic equations where the matrix elements $P_{n}$ and $Q_{n, m}$ are known (Eqs. A.26 and A.27):

$$
([\mathbb{I}]-[\mathbb{Q}])[\xi]=p_{\text {inc }}[\mathbb{P}]+[\mathbb{M}]
$$

$\mathbb{I}$ denoting the identity matrix and $\mathbb{M}$ being a column vector of elements $M_{n}$ given in Eq. (A.28).

\subsection{Rigid one-dimensional flat beam suspended at both ends on non-rigid walls (spring-like boundaries)}

The differential equation which governs the uniform amplitude of the displacement field $\eta(t)$ (positive when directed along the $y$-axis) of the rigid planar beam of mass $M$, attach at both ends $x=0$ and $x=L$ to spring-like boundaries 
of same stiffness $K_{c}$ and damping coefficient (mechanical resistance) $R_{c}$, driven by the incident uniform time-periodic acoustic pressure $p_{\text {inc }}$, can be written as:

$$
\left[-\omega^{2} M+i \omega R_{c}+K_{c}\right] \eta=b\left[\int_{0}^{L} p_{\text {gap }}(x) d x-L p_{\text {inc }}\right]-2 \int_{0}^{L}\left[\frac{d F_{s, y}(x)}{d x}\right] d x,
$$

where the second term in the right hand side of the equation, descriptive of the lateral slits (Eq. (7)), is given by

$$
\int_{0}^{L}\left[\frac{d F_{s, y}(x)}{d x}\right] d x=-\frac{1}{2} K_{v, s} h_{s}\left[L p_{\mathrm{inc}}-\int_{0}^{L} p_{\mathrm{gap}}(x) d x\right]+i \omega \eta \Pi_{s} L .
$$

Here, expression of $p_{\text {gap }}(x)$ differs slightly from the one given in section 2.3 because there is no slit at the end $(x=L)$. So, in a manner similar to that described in the derivation of the right-hand side of equation (18), the source function $U$ here is found to have the same form as in equation (22) except that the term involving the Dirac function $\delta(x-L)$ is removed (no slit effect at the end $x=L$ ):

$$
U=2 \frac{F_{v, s}}{F_{v, g}} \frac{h_{s}}{h_{g} h_{b} b} p_{\text {inc }}+\frac{\rho_{0} \omega^{2}}{F_{v, g} h_{g}}\left[1+\frac{h_{s}}{b} K_{v, s}\right] \eta .
$$

Then equation (27) gives readily the solution for the pressure variation $p_{\text {gap }}$ :

$$
p_{\text {gap }}(x)=\frac{U}{\chi^{2}}\left[\cos \left(\chi(x-L)-\alpha_{L}\right) \frac{\sin \left(\alpha_{0}\right)}{\sin \left(\chi L+\alpha_{0}+\alpha_{L}\right)}+\cos \left(\chi x+\alpha_{0}\right) \frac{\sin \left(\alpha_{L}\right)}{\sin \left(\chi L+\alpha_{0}+\alpha_{L}\right)}-1\right] .
$$

On substituting expressions (45) to (47) into equation (44) it is found that

$$
\eta=-p_{\mathrm{inc}} \frac{\left(b-K_{v, s} h_{s}\right)\left(L+\Upsilon_{s}\left(L-D_{0}\right)\right)}{-\omega^{2} M+i \omega\left(R_{c}+2 \Pi_{s} L\right)+K_{c}+\Gamma_{s} \chi\left(b-K_{v, s} h_{s}\right)\left(L-D_{0}\right)},
$$

where

$$
D_{0}=\frac{\sin \left(\alpha_{L}+\chi L\right) \sin \left(\alpha_{0}\right)+\sin \left(\alpha_{0}+\chi L\right) \sin \left(\alpha_{L}\right)-2 \sin \left(\alpha_{L}\right) \sin \left(\alpha_{0}\right)}{\chi \sin \left(\chi L+\alpha_{0}+\alpha_{L}\right)} .
$$

and where $\Upsilon_{s}$ and $\Gamma_{s}$ are given in equations (A.12) and (A.14).

\section{Numerical (FEM) implementation, comparison between analytical and numerical results}

The remaining of the paper presents: -i/ the analytical results obtained from using the solutions presented above, providing the mean displacement of the planar beams, which is the primarily desired descriptor that can be used further to provide sensitivity, input impedance, and transfer function of the device, -ii/ comparisons with results obtained from the numerical implementation presented below. Comparison with experimental results available in the literature is not really possible because both the devices and/or the parameters of interest always differ from each other on many points. At the most, one could say that the comparisons of our results with the experimental results on the dynamic response of a cantilever in air near a solid wall [17, Fig. 5 for example] show a qualitative agreement (same order of magnitude for the amplitudes of oscillations and successive resonance frequencies).

Several numerical implementations (FEM) taking into account the effects of thermal and viscous boundary layers in thermoviscous fluids have been proposed in the literature $[18,19,20]$. The numerical implementation handled herein, against which the approximate analytical results can be tested, relies on 3-D simulation performed using the software Comsol Multiphysics, version 5.1. The linear formulation used to perform the numerical modelling in the slits, fluid gap, and peripheral cavities is based upon the following coupled equations involving the particle velocity $\vec{v}$, the temperature variation $\tau$ and the acoustic pressure $p[20,21]$. 


$$
\begin{aligned}
i \omega \rho_{0} \mathbf{v}-\nabla \cdot\left[-p \mathbb{I}+\mu\left(\nabla \mathbf{v}+\nabla \mathbf{v}^{\mathrm{T}}\right)-\left(\frac{2}{3} \mu-\mu_{B}\right)(\nabla \cdot \mathbf{v}) \mathbb{I}\right] & =\mathbf{0} \\
i \omega \rho+\rho_{0} \nabla \cdot \mathbf{v} & =0 \\
i \omega\left(\rho_{0} C_{P} \tau-p T_{0} \alpha_{0}\right)+\nabla \cdot\left(-\lambda_{h} \nabla \tau\right) & =0
\end{aligned}
$$

where

$$
\begin{aligned}
\rho & =\rho_{0}\left(\beta_{T} p-\alpha_{0} \tau\right), \\
\alpha_{0} & =-\left.\frac{1}{\rho_{0}} \frac{\partial \rho_{0}}{\partial T_{0}}\right|_{P_{0}}, \\
\beta_{T} & =\left.\frac{1}{\rho_{0}} \frac{\partial \rho_{0}}{\partial P_{0}}\right|_{T_{0}},
\end{aligned}
$$

where the dependence of the equilibrium density $\rho_{0}$ on the static pressure $P_{0}$ and the static temperature $T_{0}$ is given by $\rho_{0}\left(P_{0}, T_{0}\right)=0.02897 P_{0} /\left(8.314 T_{0}\right)[21]$, and where the particle velocity and the temperature variation are subjected to Dirichlet conditions on the rigid isothermal boundaries $(\mathbf{v}=\mathbf{0}$ and $\tau=0)$.

The numerical modeling of the motion of the beams is based on the classical linear formulation [22]

$$
\begin{aligned}
-\omega^{2} \mathbf{u} & =\nabla \sigma \\
\sigma & =\mathrm{C}: \varepsilon, \\
\varepsilon & =\frac{1}{2}\left([\nabla \mathbf{u}]^{T}+\nabla \mathbf{u}\right),
\end{aligned}
$$

where $\mathbf{u}$ is the displacement vector, $\sigma$ and $\varepsilon$ are the stress and strain tensor respectively, ':' stands for a double contraction over two indices $\left(\sigma_{i j}=\mathrm{C}_{i j k l} \varepsilon_{k l}\right)$, C being the elasticity tensor whose elements depend on the Young's modulus $E$ and Poisson's ratio $\nu$ [22] (linear elastic isotropic material). At the interface between the acoustical and the mechanical domains, the continuity of the velocity $(\mathbf{v}=i \omega \mathbf{u})$ along with the isothermal condition $(\tau=0)$ are applied. The time-periodic adiabatic acoustic pressure field with amplitude of 1 Pa, representing the uniform excitation at the input of the device, and the properties of the thermoviscous fluid (air in this case) given in Table 1 are used in both analytical and numerical modelling. The analytical displacement of the beam $\eta$ is compared with the y-component (according to Fig. 2) of the numerically calculated displacement vector $\mathbf{u}$.

Figs. 5, 6, 7 display the amplitude and the phase of the mean displacement of silicon microbeams $\left(\rho_{b}=\right.$ $\left.2329 \mathrm{~kg} / \mathrm{m}^{3}\right)$, in using the values of the parameters given in table 2, as a function of the frequency in the frequency range $(100 \mathrm{~Hz}, 1 \mathrm{MHz})$. These beams are called: beam A - a rigid one-dimensional planar beam attached to one end of a flat spring; beam B - a thin elastic one-dimensional flat beam clamped at one end and free at the other end; and beam $\mathrm{C}$ - a rigid one-dimensional flat beam suspended at both ends on non-rigid walls (spring-like boundaries). In these results small cavities (with dimensions being chosen the same as those of the gap) are set at both ends $(x=0, x=L)$ of the beam B, at one end of the beam $\mathrm{C}$, and at the free end $(x=L)$ of the beam A.

The minor differences highlighted by the comparison between the analytical results (full line) and the numerical FEM results (circular marks) validate the relevance of the theoretical modelling even though several assumptions have been retained. The most important of these assumptions would be that used for describing the behaviour of the very small slits, the effects of discontinuity being omitted.

\section{Conclusion}

The original motivation for this study was to characterise the motion of three kinds of planar micro-beams (one dimensional plate) when a reaction pressure on this plate is provided by the motion of a fluid layer in a backing cavity, the acoustic field in this fluid layer being both subjected to appropriate boundary conditions and coupled 

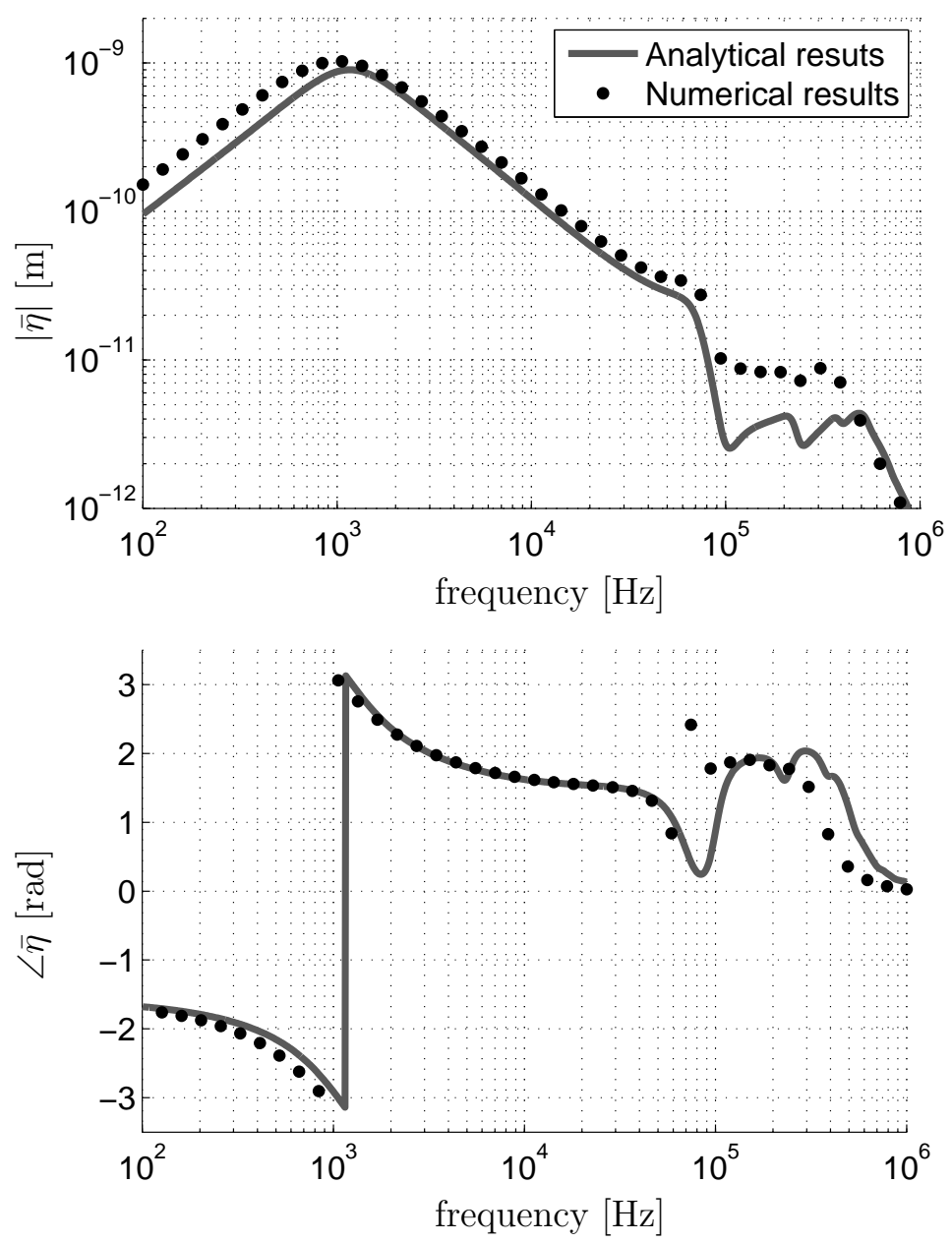

Figure 5. Beam A: Rigid one-dimensional planar beam attached to one end of a flat spring of negligible mass.

\begin{tabular}{l|c|c} 
Parameter & Value & Unit \\
\hline Static pressure $P_{0}$ & 101330 & $\mathrm{~Pa}$ \\
Static temperature $T_{0}$ & 293.15 & $\mathrm{~K}$ \\
Density $\rho_{0}$ & 1.204 & $\mathrm{~kg} \mathrm{~m}^{-1}$ \\
Adiabatic speed of sound $c_{0}$ & 343.2 & $\mathrm{~m} \mathrm{~s}^{-1}$ \\
Shear dynamic viscosity $\mu$ & $1.814 \times 10^{-5}$ & $\mathrm{~Pa} \mathrm{~s}$ \\
Bulk dynamic viscosity $\mu_{B}$ & $1.088 \times 10^{-5}$ & $\mathrm{~Pa} \mathrm{~s}$ \\
Thermal conductivity $\lambda_{h}$ & $25.77 \times 10^{-3}$ & $\mathrm{~W} \mathrm{~m} \mathrm{~K}^{-1}$ \\
Ratio of specific heats $\gamma$ & 1.400 & - \\
Specific heat coefficient $C_{P}$ & 1005 & $\mathrm{~J} \mathrm{~kg}^{-1} \mathrm{~K}^{-1}$
\end{tabular}

Table 1. Parameters of the thermoviscous fluid. 

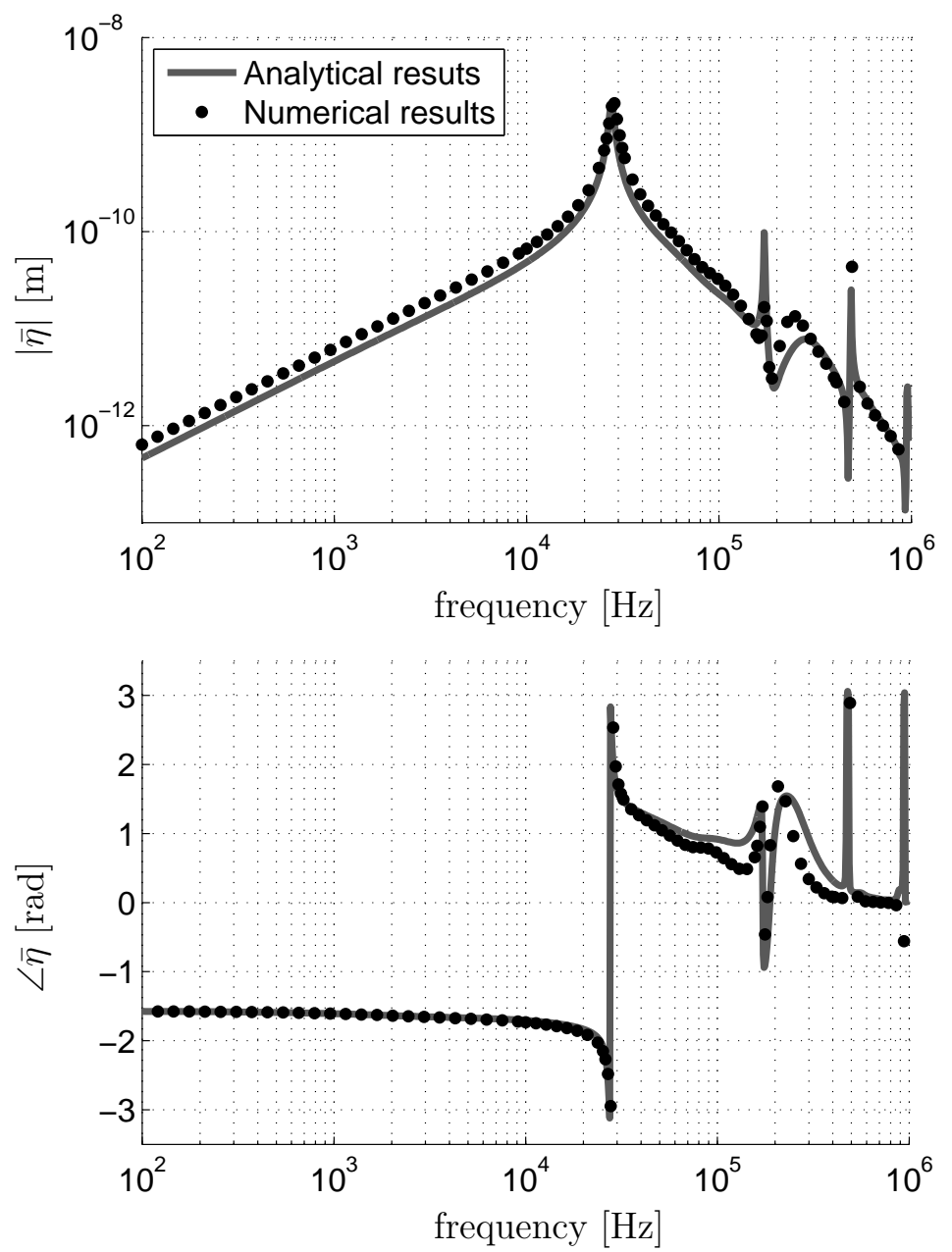

Figure 6. Beam B: Thin elastic one-dimensional flat beam clamped at one end and free at the other end. 

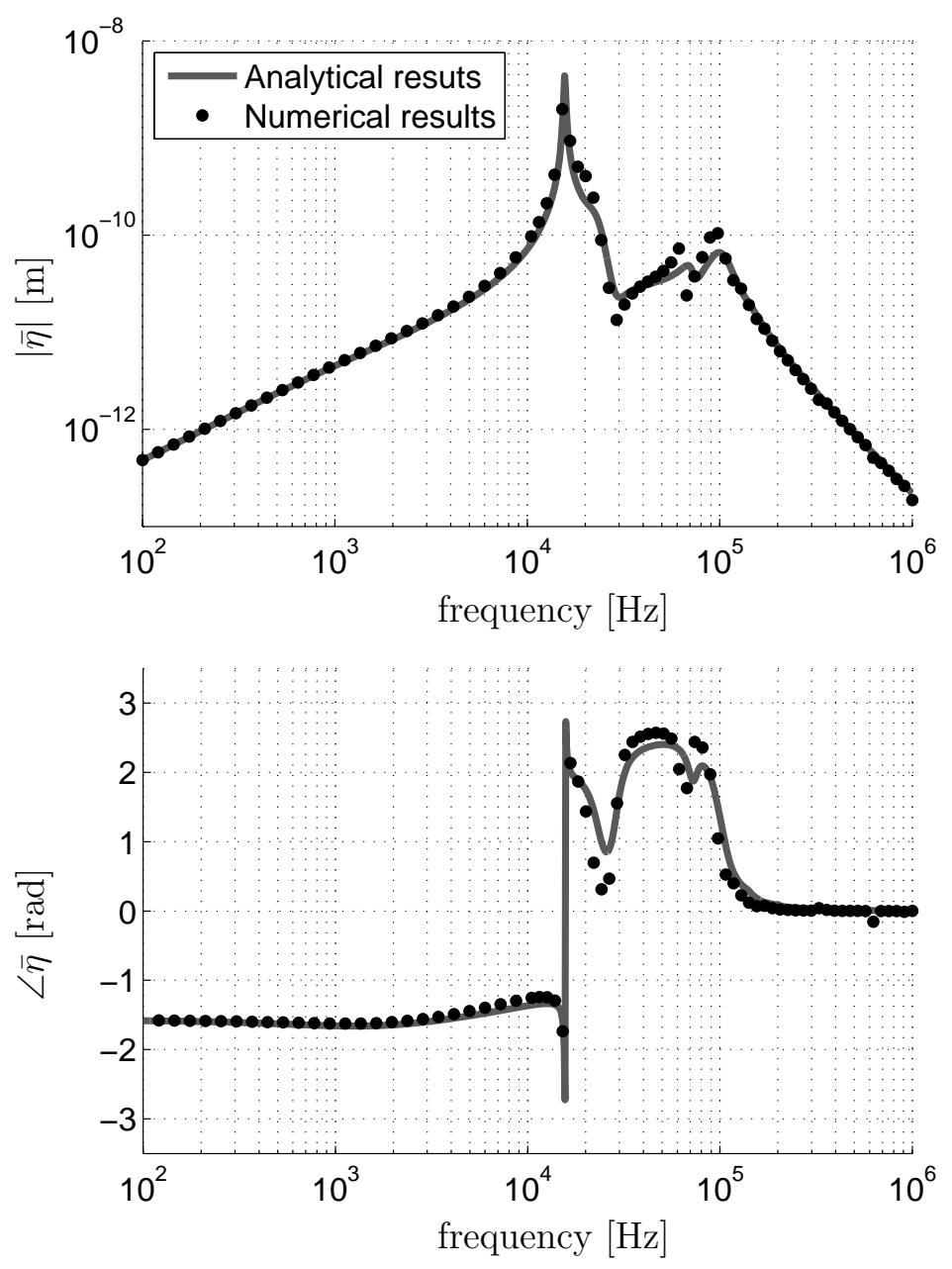

Figure 7. Beam C: Rigid one-dimensional flat beam suspended at both ends on non-rigid walls (spring-like boundaries) 
to the incident pressure field through slits at the periphery of the beam. A general approach, based on the exact description of the strong coupling between the moving structure and the fluid layer, is used for the analysis of the motion of both the fluid layer and the beam (it includes viscous and thermal effects which depend strongly on the thickness profile of the fluid gap and the slits). The solution of the problem of two coupled differential and integral equations describing respectively the displacement field of the beam and the pressure field in the backing cavity is derived.

The main contribution of the paper is thus a systematic framework to estimate accurately the motion or the shape of the displacement field of the micro-beam for a given external pressure field, accounting for the complex coupling mentioned above. Further frequency dependent quantities may then be obtained, namely the sensitivity of these kinds of device when they are used as receivers, and the input impedance or the acoustic transfer function when they are used as passive components. The system considered here is described with a refinement that should be consistent with the requirements of the devices which could appear in a near future, when miniaturisation (using MEMS technique), when very large bandwidth (up to $1 \mathrm{MHz}$, even more), and when precise analysis or measurement, among others, would be required. Actually, in the lower frequency range (up to $20 \mathrm{kHz}$ for example) and for small dimensions, lumped circuit elements that could be derived assuming appropriate approximations would provide accurate modelling in the usual situations (such modelling is beyond the scope of this paper).

The theoretical results presented in this paper convey an interpretation of physical phenomena, giving the role played by several parameters as mentioned above. These results for the acoustic field in the backing cavity and the motion or the shape profile of the beam are obtained in a straightforward manner, irrespective of frequency. A numerical implementation (FEM) has been handled which permits to validate the analytical results. Thus finally, requirements that have to be taken into account in the design of such vibrating devices could be addressed with a very good accuracy, using the theoretical results obtained in this work.

\section{Acknowledgement}

This research was funded by the Region Pays de la Loire within the Le Mans Acoustic Project and partially supported by the Grant Agency of the Czech Technical University in Prague, grant No. SGS15/226/OHK2/3T/16. The authors are indebted to Dr. Nicolas Joly from LAUM for substantial help in providing numerical facilities. They would like to express their appreciation to Dr. François Fohr from CTTM for helpful discussions.

\begin{tabular}{c||c|c|c|c|c|c||c|c||c|c||c|c} 
& $\begin{array}{c}L \\
{[\mathrm{~mm}]}\end{array}$ & $\begin{array}{c}b \\
{[\mathrm{~mm}]}\end{array}$ & $\begin{array}{c}h_{b} \\
{[\mu \mathrm{m}]}\end{array}$ & $\begin{array}{c}h_{g} \\
{[\mu \mathrm{m}]}\end{array}$ & $\begin{array}{c}h_{s} \\
{[\mu \mathrm{m}]}\end{array}$ & $\begin{array}{c}h_{L} \\
{[\mu \mathrm{m}]}\end{array}$ & $\begin{array}{c}C_{b} \\
{[\mu \mathrm{Nm}]}\end{array}$ & $\begin{array}{c}D_{b} \\
{[\mathrm{Ns}]}\end{array}$ & $\begin{array}{c}E \\
{[\mathrm{GPa}]}\end{array}$ & $\begin{array}{c}\nu \\
{[-]}\end{array}$ & $\begin{array}{c}K_{c} \\
{[\mathrm{~N} / \mathrm{m}]}\end{array}$ & $\begin{array}{c}R_{c} \\
{[\mathrm{~N} \mathrm{~s} / \mathrm{m}]}\end{array}$ \\
\hline beam A & 1.0 & 0.2 & 20 & 50 & 10 & 10 & 0.125 & 0 & - & - & - & - \\
beam B & 1.0 & 0.4 & 20 & 100 & 10 & 10 & - & - & 170 & 0.28 & - & - \\
beam C & 3.0 & 1.0 & 50 & 150 & 30 & 30 & - & - & - & - & 3.4 & 0
\end{tabular}

Table 2. Parameters of the three beams used in the numerical comparisons. Beam A is a rigid one-dimensional planar beam attached to one end of a flat spring, beam B is a thin elastic one-dimensional flat beam clamped at one end and free at the other end, and beam $\mathrm{C}$ is a rigid one-dimensional flat beam suspended at both ends on non-rigid walls (spring-like boundaries). 


\section{Appendix}

\section{A.1 Expression of $p_{\text {gap }}$ (Eq. 27) associated with the oscillations of the rigid beam attached to one end of a} flat spring (section 3.1)

The pressure variation $p_{\text {gap }}$ in the backing cavity (fluid-gap) admits the integral representation (27) wherein $G\left(x, x_{0}\right)$ denotes the Green function (25) and $U\left(x_{0}\right)$ is proportional to the total input volume velocity (22). The function $U\left(x_{0}\right)$ involves, among others, the mean value of the velocity profile $\bar{v}_{L, y}$ (Eq. 14) in the slit set at $x=L$ and the displacement field of the planar beam $\eta(x)=x \theta$. These last functions depend respectively on $p_{\text {gap }}(L)$ and $\int_{0}^{L} p_{\text {gap }}(x) x d x$, which are independent of the integration variable $x$. Therefore, the integrands in Eq. (27) contains only simple trigonometric functions of $x$ that can be integrated readily. After effecting these integrations, it follows that $p_{\text {gap }}(x)$ can be expressed as a linear function of $p_{\text {gap }}(L), p_{\text {inc }}$ and $\theta$, which leads readily to, when writing $x=L$,

$$
p_{\text {gap }}(L)=-B_{1} p_{\text {inc }}-B_{2} \theta,
$$

then in turn, after relying on (A.1), to

$$
p_{\text {gap }}(x)=C_{1}(x) p_{\text {gap }}(L)-C_{2}(x) p_{\text {inc }}-C_{3}(x) \theta,
$$

and finally to

$$
\int_{0}^{L} p_{\text {gap }}(x) x d x=A_{1} p_{\text {gap }}(L)-A_{2} p_{\text {inc }}-A_{3} \theta
$$

where

$$
\begin{gathered}
A_{1}=\frac{1}{\chi^{2}} \frac{\Upsilon_{L} \cos \left(\alpha_{L}\right)}{\sin \left(\chi L+\alpha_{0}+\alpha_{L}\right)}\left[\chi L \sin \left(\chi L+\alpha_{0}\right)+\cos \left(\chi L+\alpha_{0}\right)-\cos \left(\alpha_{0}\right)\right], \\
A_{2}=\frac{1}{\chi^{2}}\left\{\frac{\Upsilon_{L} \cos \left(\alpha_{L}\right)-\Upsilon_{s} \sin \left(\alpha_{L}\right)}{\sin \left(\chi L+\alpha_{0}+\alpha_{L}\right)}\left[\chi L \sin \left(\chi L+\alpha_{0}\right)+\cos \left(\chi L+\alpha_{0}\right)-\cos \left(\alpha_{0}\right)\right]\right. \\
\left.+\Upsilon_{s}\left[\frac{(\chi L)^{2}}{2}+\sin \left(\alpha_{0}\right) \frac{\chi L \sin \left(\alpha_{L}\right)+\cos \left(\chi L+\alpha_{L}\right)-\cos \left(\alpha_{L}\right)}{\sin \left(\chi L+\alpha_{0}+\alpha_{L}\right)}\right]\right\}, \\
A_{3}=\frac{1}{\chi^{2}}\left\{\frac{\Gamma_{L} \chi L \cos \left(\alpha_{L}\right)+\Gamma_{s}\left(\cos \left(\alpha_{L}\right)-\chi L \sin \left(\alpha_{L}\right)\right)}{\sin \left(\chi L+\alpha_{0}+\alpha_{L}\right)}\left(\chi L \sin \left(\chi L+\alpha_{0}\right)+\cos \left(\chi L+\alpha_{0}\right)-\cos \left(\alpha_{0}\right)\right)\right. \\
\left.+\Gamma_{s}\left[\frac{(\chi L)^{3}}{3}+\cos \left(\alpha_{0}\right) \frac{\chi L \sin \left(\alpha_{L}\right)+\cos \left(\chi L+\alpha_{L}\right)-\cos \left(\alpha_{L}\right)}{\sin \left(\chi L+\alpha_{0}+\alpha_{L}\right)}\right]\right\} . \\
B_{1}=\frac{\Upsilon_{L} \cos \left(\chi L+\alpha_{0}\right)+\Upsilon_{s}\left(\sin \left(\chi L+\alpha_{0}\right)-\sin \left(\alpha_{0}\right)\right)}{\frac{\sin \left(\chi L+\alpha_{0}+\alpha_{L}\right)}{\cos \left(\alpha_{L}\right)}-\Upsilon_{L} \cos \left(\chi L+\alpha_{0}\right)}, \\
B_{2}=\frac{\chi \Gamma_{L} L \cos \left(\chi L+\alpha_{0}\right)+\Gamma_{s}\left(\chi L \sin \left(\chi L+\alpha_{0}\right)+\cos \left(\chi L+\alpha_{0}\right)-\cos \left(\alpha_{0}\right)\right)}{\frac{\sin \left(\chi L+\alpha_{0}+\alpha_{L}\right)}{\cos \left(\alpha_{L}\right)}-\Upsilon_{L} \cos \left(\chi L+\alpha_{0}\right)},
\end{gathered}
$$

and

$$
C_{1}(x)=\frac{\cos \left(\chi x+\alpha_{0}\right) \cos \left(\alpha_{L}\right)}{\sin \left(\chi L+\alpha_{0}+\alpha_{L}\right)} \Upsilon_{L},
$$




$$
\begin{aligned}
C_{2}(x)= & C_{1}(x)+\Upsilon_{s}\left[1-\frac{\cos \left(\chi(x-L)-\alpha_{L}\right) \sin \left(\alpha_{0}\right)+\cos \left(\chi x+\alpha_{0}\right) \sin \left(\alpha_{L}\right)}{\sin \left(\chi L+\alpha_{0}+\alpha_{L}\right)}\right], \\
C_{3}(x)= & \frac{\Gamma_{s}+\Gamma_{L} \chi L}{\sin \left(\chi L+\alpha_{0}+\alpha_{L}\right)} \cos \left(\chi x+\alpha_{0}\right) \cos \left(\alpha_{L}\right) \\
& +\Gamma_{s}\left[\chi x-\frac{\cos \left(\alpha_{0}\right) \cos \left(\chi(x-L)-\alpha_{L}\right)+\chi L \sin \left(\alpha_{L}\right) \cos \left(\chi x+\alpha_{0}\right)}{\sin \left(\chi L+\alpha_{0}+\alpha_{L}\right)}\right],
\end{aligned}
$$

with (additional parameters)

$$
\begin{gathered}
\Upsilon_{s}=\frac{2}{\chi^{2}} \frac{F_{v, s}}{F_{v, g}} \frac{h_{s}}{h_{g} h_{b} b}, \\
\Upsilon_{L}=\frac{1}{\chi} \frac{F_{v, L}}{F_{v, g}} \frac{h_{L}}{h_{g} h_{b}},
\end{gathered}
$$

and (pressure dimension)

$$
\begin{gathered}
\Gamma_{s}=\frac{\rho_{0} \omega^{2}}{\chi^{3} F_{v, g} h_{g}}\left[1+\frac{h_{s}}{b} K_{v, s}\right], \\
\Gamma_{L}=\frac{\rho_{0} \omega^{2}}{\chi^{2}} \frac{K_{v, L}}{2 F_{v, g}} \frac{h_{L}}{h_{g}} .
\end{gathered}
$$

\section{A.2 Expression of $p_{\text {gap }}$ (Eq. 27) associated with the oscillations of the elastic beam clamped to one end (section 3.2)}

The procedure presented in this appendix is similar to the one presented in Appendix A.1, apart from the displacement field of the planar beam $\eta(x)=x \theta$ which is replaced by the modal expression $\eta(x)=\sum_{m} \xi_{m} \psi_{m}(x)$ (41). Therefore, the integrands in equation (27) contain either simple trigonometric functions or the product of two trigonometric functions that can be integrated readily. After effecting these integrations, it follows that $p_{\text {gap }}(x)$ can be expressed as a linear function of $p_{\text {gap }}(L), p_{\text {inc }}$, and an expansion involving the unknown modal coefficients $\xi_{m}$, which leads readily to, when writing $x=L$,

$$
p_{\text {gap }}(L)=p_{\text {inc }} \frac{\Phi_{2}(L)}{1-\Phi_{1}(L)}+\sum_{m} \xi_{m} \frac{\varphi_{m}(L)}{1-\Phi_{1}(L)},
$$

then to the pressure variation in the backing cavity

$$
p_{\text {gap }}(x)=p_{\text {inc }}\left[\Phi_{2}(x)+\Phi_{2}(L) \frac{\Phi_{1}(x)}{1-\Phi_{1}(L)}\right]+\sum_{m} \xi_{m}\left[\varphi_{m}(x)+\varphi_{m}(L) \frac{\Phi_{1}(x)}{1-\Phi_{1}(L)}\right],
$$

where

$$
\begin{gathered}
\Phi_{1}(x)=\frac{\Upsilon_{L} \cos \left(\alpha_{L}\right)}{\sin \left(\chi L+\alpha_{0}+\alpha_{L}\right)} \cos \left(\chi x+\alpha_{0}\right), \\
\Phi_{2}(x)=\frac{\Upsilon_{s} \sin \left(\alpha_{0}\right)}{\sin \left(\chi L+\alpha_{0}+\alpha_{L}\right)} \cos \left(\chi(x-L)-\alpha_{L}\right)+\frac{\Upsilon_{s} \sin \left(\alpha_{L}\right)-\Upsilon_{L} \cos \left(\alpha_{L}\right)}{\sin \left(\chi L+\alpha_{0}+\alpha_{L}\right)} \cos \left(\chi x+\alpha_{0}\right)-\Upsilon_{s},
\end{gathered}
$$

and

$$
\begin{aligned}
\varphi_{m}(x)= & \frac{2 \chi}{\sqrt{L}} \frac{\cos \left(\chi(x-L)-\alpha_{L}\right) \chi \Gamma_{s} K_{m}^{2} W_{m}^{+}\left(\alpha_{0}\right)-(-1)^{m} \cos \left(\chi x+\alpha_{0}\right)\left(\Gamma_{s} \chi^{3} W_{m}^{+}\left(\alpha_{L}\right)-\Gamma_{L} \cos \left(\alpha_{L}\right)\right)}{\sin \left(\chi L+\alpha_{0}+\alpha_{L}\right)} \\
& +\frac{\chi^{3} \Gamma_{s}}{\sqrt{L}}\left(\frac{\cosh \left(K_{m} x\right)+\beta_{m} \sinh \left(K_{m} x\right)}{\chi^{2}+K_{m}^{2}}-\frac{\cos \left(K_{m} x\right)+\beta_{m} \sin \left(K_{m} x\right)}{\chi^{2}-K_{m}^{2}}\right),
\end{aligned}
$$

where

$$
W_{m}^{ \pm}(\alpha)=\frac{\chi \sin (\alpha) \pm \beta_{m} K_{m} \cos (\alpha)}{\chi^{4}-K_{m}^{4}}
$$


Note that these last expressions has been obtained after accounting for the following relationships

$$
\begin{gathered}
\frac{\sin \left(K_{m} L\right) \sinh \left(K_{m} L\right)}{\cos \left(K_{m} L\right)+\cosh \left(K_{m} L\right)}=(-1)^{m}, \\
\frac{\sin \left(K_{m} L\right) \sinh \left(K_{m} L\right)}{\sin \left(K_{m} L\right)+\sinh \left(K_{m} L\right)}=-(-1)^{m} \beta_{m},
\end{gathered}
$$

that readily arise from equation $\cos \left(K_{m} L\right) \cosh \left(K_{m} L\right)=-1$ whose the roots can be written as

$$
K_{m} L=(2 m+1) \frac{\pi}{2}+\left(-\varepsilon_{m}\right)^{m},
$$

with $\varepsilon_{0} \cong 0.2 \frac{\pi}{2}$ and $0<\varepsilon_{m} \ll \frac{\pi}{2}, m=1,2,3, \ldots$

Finally, utilizing (A.16) and the expressions readily obtained for the integrals which appear in equation (42), namely $\int_{0}^{L} p_{\text {gap }}(x) \psi_{m}(x) d x$ and $\int_{0}^{L} \psi_{m}(x) d x$, the unknown coefficients $\xi_{m}$ are, therefore, solutions of the linear set of algebraic equations

$$
\xi_{n}=p_{\text {inc }} P_{n}+\sum_{m} \xi_{m} Q_{n, m}+M_{n},
$$

that can be written in the form of the square matrix equation (43), where

$$
\begin{gathered}
P_{n}=\left(b-K_{v, s} h_{s}\right)\left(\int_{0}^{L} \Phi_{2}(x) \Psi_{n}(x) d x+\int_{0}^{L} \Phi_{1}(x) \Psi_{n}(x) d x \frac{\Phi_{2}(L)}{1-\Phi_{1}(L)}-\frac{2 \beta_{n}}{K_{n} \sqrt{L}}\right) \\
-\frac{K_{v, L} h_{L} b}{\sqrt{L}}(-1)^{n}\left(1-\frac{\Phi_{2}(L)}{1-\Phi_{1}(L)}\right), \\
Q_{n, m}=\left(b-K_{v, s} h_{s}\right)\left(\int_{0}^{L} \varphi_{m}(x) \Psi_{n}(x) d x+\int_{0}^{L} \Phi_{1}(x) \Psi_{n}(x) d x \frac{\varphi_{m}(L)}{1-\Phi_{1}(L)}\right) \\
+(-1)^{n} \frac{K_{v, L} h_{L} b}{\sqrt{L}} \frac{\varphi_{m}(L)}{1-\Phi_{1}(L)}-(-1)^{m+n} j \omega \frac{4 \Pi_{L} b}{L},
\end{gathered}
$$

and

$$
M_{n}=\frac{-(-1)^{n} \frac{2}{\sqrt{L}} \omega^{2} M_{\mathrm{add}}}{E I K_{n}^{4}-\omega^{2} \mu_{L}+i \omega 2 \Pi_{s}}
$$

where

$$
\begin{aligned}
\int_{0}^{L} \Phi_{1}(x) & \Psi_{n}(x) d x=-\chi \Upsilon_{L} \cos \left(\alpha_{L}\right) \frac{2}{\sqrt{L}} V_{n}^{-}\left(\chi L+\alpha_{0}, \alpha_{0}\right) \\
\int_{0}^{L} \Phi_{2}(x) \Psi_{n}(x) d x= & \frac{2 \chi}{\sqrt{L}}\left[\Upsilon_{s} \sin \left(\alpha_{0}\right) V_{n}^{+}\left(\alpha_{L}, \chi L+\alpha_{L}\right)\right. \\
& \left.+\left(\Upsilon_{L} \cos \left(\alpha_{L}\right)-\Upsilon_{s} \sin \left(\alpha_{L}\right)\right) V_{n}^{-}\left(\chi L+\alpha_{0}, \alpha_{0}\right)-\frac{\Upsilon_{s}}{\chi} \frac{\beta_{n}}{K_{n}}\right]
\end{aligned}
$$

and

$$
\begin{aligned}
\int_{0}^{L} \varphi_{m}(x) \Psi_{n}(x) d x= & \frac{4 \chi^{2}}{L}\left[(-1)^{m}\left(\Gamma_{s} \chi^{3} W_{m}^{+}\left(\alpha_{L}\right)-\Gamma_{L} \cos \left(\alpha_{L}\right)\right) V_{n}^{-}\left(\chi L+\alpha_{0}, \alpha_{0}\right)\right. \\
& \left.+\Gamma_{s} \chi K_{m}^{2} W_{m}^{+}\left(\alpha_{0}\right) V_{n}^{+}\left(\alpha_{L}, \chi L+\alpha_{L}\right)\right]-\frac{\Gamma_{s} \chi^{3}}{\chi^{4}-K_{m}^{4}} X_{m, n}
\end{aligned}
$$

with

$$
X_{m, n}= \begin{cases}\frac{4}{L} K_{m}^{2}\left(\beta_{m} K_{m}-\beta_{n} K_{n}\right) \frac{(-1)^{m+n} K_{m}^{2}+K_{n}^{2}}{K_{m}^{4}-K_{n}^{4}}, & m \neq n \\ \beta_{m} K_{m}\left(\frac{2}{L}+\beta_{m} K_{m}\right)+\chi^{2}, & m=n,\end{cases}
$$


and

$$
V_{n}^{ \pm}\left(\alpha_{x}, \alpha_{y}\right)=\frac{(-1)^{n} \chi^{2} W_{n}^{ \pm}\left(\alpha_{x}\right)+K_{n}^{2} W_{n}^{\mp}\left(\alpha_{y}\right)}{\chi \sin \left(\chi L+\alpha_{0}+\alpha_{L}\right)}
$$

Reporting these solutions in equation (41) yields directly the sought-after solution for the displacement field of the beam $\eta(x)$.

\section{A.3 On the orthogonality and the completeness of the eigenfunctions $\psi_{m}(x)$}

Equation (37) $\left[\frac{\partial^{4}}{\partial x^{4}}-K_{m}^{4}\right] \psi_{m}(x)=0$ leads readily to the following equation:

$$
\psi_{m}(x) \frac{\partial^{4}}{\partial x^{4}} \psi_{n}(x)-\psi_{n}(x) \frac{\partial^{4}}{\partial x^{4}} \psi_{m}(x)=\left[K_{n}^{4}-K_{m}^{4}\right] \psi_{m}(x) \psi_{n}(x),
$$

which can be written as

$$
\begin{gathered}
\frac{\partial}{\partial x}\left\{\psi_{m}(x) \frac{\partial^{3}}{\partial x^{3}} \psi_{n}(x)-\frac{\partial}{\partial x} \psi_{m}(x) \frac{\partial^{2}}{\partial x^{2}} \psi_{n}(x)+\frac{\partial^{2}}{\partial x^{2}} \psi_{m}(x) \frac{\partial}{\partial x} \psi_{n}(x)-\psi_{n}(x) \frac{\partial^{3}}{\partial x^{3}} \psi_{m}(x)\right\} \\
=\left[K_{n}^{4}-K_{m}^{4}\right] \psi_{m}(x) \psi_{n}(x)
\end{gathered}
$$

Integrating over the domain $x \in(0, L)$ and accounting for the boundary conditions (35) and (36) shows straightforwardly the orthogonality property of the eigenfunctions:

$$
0=\left[K_{n}^{4}-K_{m}^{4}\right] \psi_{m}(x) \psi_{n}(x)
$$

Note that this result can be verified by substituting expression (4) for $\psi_{m}(x)$ into the right hand side of this equation and by accounting for the eigen-equation $\cos \left(K_{m} L\right) \cosh \left(K_{m} L\right)=-1$.

When $m=n$, what result is $\int_{0}^{L} \psi_{m}^{2}(x) d x=1$ due to the normalisation constant $\sqrt{1 / L}$ chosen (Eq. 4). Finally, considering that these orthonormal eigenfunctions are merely a generalization of the familiar functions of Fourier series, there is no doubt that their completeness is well established.

\section{A.4 On the thermal behaviour of the fluid gap}

Concerning the thermal behaviour of the fluid-film, relaxation phenomena occur that involve complex compressibility coefficient and energy dissipation [14]. Fig. 8 shows readily that this thermal behaviour should be accounted for in the frequency range considered here (up to $1 \mathrm{MHz}$ ) when the air-gap thickness is of the order of magnitude of 100 micrometers. This figure represents the modulus and the phase of the factor $\left[\gamma-(\gamma-1) F_{h, g}\right]$ in Eq. $(19)$, which tends to the specific heat ratio $\gamma$ at the lower frequencies leading to the isothermal speed of sound $c_{0} / \sqrt{\gamma}$ and tends to 1 at the highest frequencies leading to the adiabatic speed of sound $c_{0}$. This quantity is approximately real at these limiting frequencies and complex between them. 

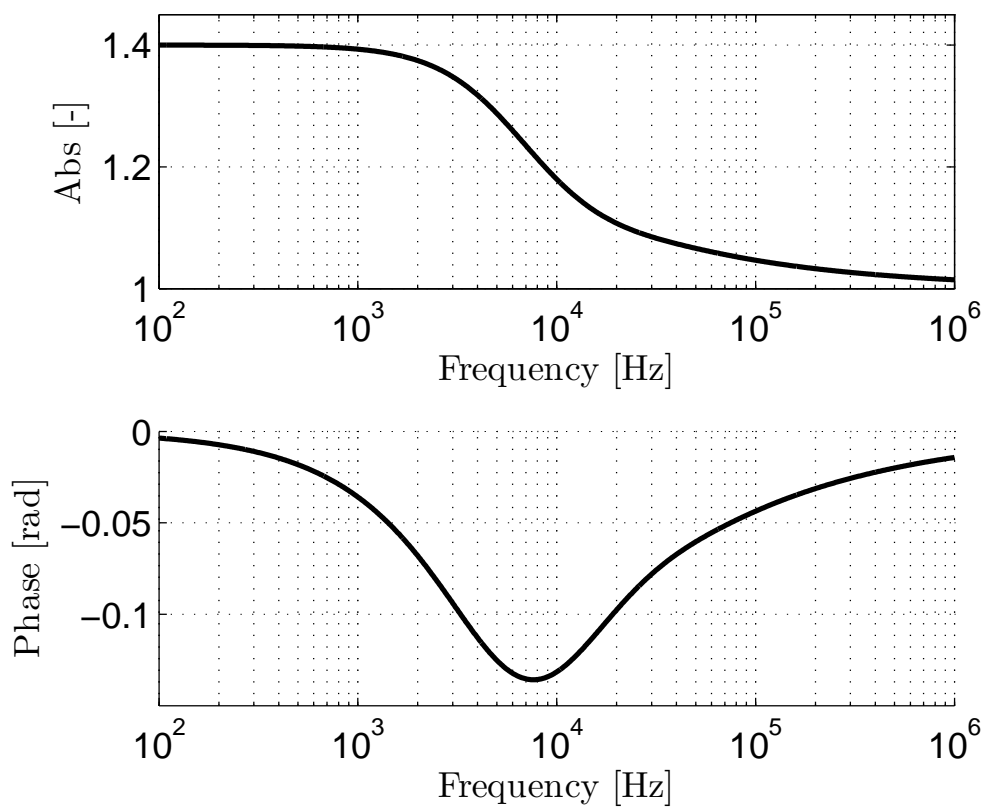

Figure 8. Modulus and phase of the factor $\left[\gamma-(\gamma-1) F_{h, g}\right]$ as a function of the frequency.

\section{References}

[1] G. M. Sessler, Silicon microphones, Journal of the Audio Engineering Society 44 (1/2) (1996) 16-22.

[2] C. Guianvarc'h, R. M. Gavioso, G. Benedetto, L. Pitre, M. Bruneau, Characterization of condenser microphones under different environmental conditions for accurate speed of sound measurements with acoustic resonators, Review of Scientific Instruments 80 (7) (2009) 074901.

[3] M. Bruneau, A.-M. Bruneau, P. Dupire, A model for rectangular miniaturized microphones, Acta acustica 3 (3) (1995) 275-282.

[4] P. Honzík, M. Bruneau, Acoustic fields in thin fluid layers between vibrating walls and rigid boundaries: integral method, Acta Acustica united with Acustica 101 (4) (2015) 859-862.

[5] S. Fukui, R. Kaneko, Analysis of ultra-thin gas film lubrication based on linearized boltzmann equation: First report - derivation of a generalized lubrication equation including thermal creep flow, Journal of Tribology 110 (1988) 253-261.

[6] L. Desvillettes, S. Lorenzani, Sound wave resonances in micro-electro-mechanical systems devices vibrating at high frequencies according to the kinetic theory of gases, Physics of Fluids 24 (9) (2012) 1-24.

[7] L. Wu, J. M. Reese, Y. Zhang, Oscillatory rarefied gas flow inside rectangular cavities, Journal of Fluid Mechanics 748 (2014) 350-367.

[8] T. Veijola, H. Kuisma, J. Lahdenperä, T. Ryhänen, Equivalent-circuit model of the squeezed gas film in a silicon accelerometer, Sensors and Actuators A: Physical 48 (3) (1995) 239-248.

[9] A. H. Nayfeh, M. I. Younis, A new approach to the modeling and simulation of flexible microstructures under the effect of squeeze-film damping, Journal of Micromechanics and Microengineering 14 (2) (2003) 170.

[10] T. Veijola, A. Lehtovuori, Numerical and analytical modelling of trapped gas in micromechanical squeeze-film dampers, Journal of Sound and Vibration 319 (1) (2009) 606-621. 
[11] T. Verdot, E. Redon, K. Ege, J. Czarny, C. Guianvarc'h, J.-L. Guyader, Microphone with planar nano-gauge detection: fluid-structure coupling including thermoviscous effects, Acta Acustica united with Acustica 102 (3) (2016) 517-529.

[12] R. B. Darling, C. Hivick, J. Xu, Compact analytical modeling of squeeze film damping with arbitrary venting conditions using a green's function approach, Sensors and Actuators A: Physical 70 (1) (1998) 32-41.

[13] M. Bruneau, A. Bruneau, Z. Škvor, P. Lotton, An equivalent network modelling the strong coupling between a vibrating membrane and a fluid film, Acta Acustica 2 (C5) (1994) 223-232.

[14] M. Bruneau, T. Scelo, Fundamentals of acoustics, ISTE, London, 2006.

[15] P. M. Morse, K. U. Ingard, Theoretical acoustics, Princeton University Press, 1968.

[16] A. W. Leissa, Vibration of plates, Scientific and Technical Information Division, National Aeronautics and Space Administration, 1969.

[17] T. Naik, E. K. Longmire, S. C. Mantell, Dynamic response of a cantilever in liquid near a solid wall, Sensors and Actuators A: physical 102 (3) (2003) 240-254.

[18] N. Joly, Finite element modeling of thermoviscous acoustics on adapted anisotropic meshes: Implementation of the particle velocity and temperature variation formulation, Acta acustica united with acustica 95 (1) (2009) 102-114.

[19] W. Kampinga, Y. Wijnant, A. de Boer, An efficient finite element model for viscothermal acoustics, Acta Acustica united with Acustica 97 (4) (2011) 618-631.

[20] M. J. H. Jensen, E. S. Olsen, Virtual prototyping of condenser microphones using the finite element method for detailed electric, mechanic, and acoustic characterization, in: Proceedings of Meetings on Acoustics, Vol. 19, Acoustical Society of America, 2013, p. 030039.

[21] COMSOL Multiphysics, Acoustics Module User's Guide (2015).

[22] COMSOL Multiphysics, Structural Mechanics Module User's Guide (2015). 